\title{
The Most Detailed Picture Yet of an Embedded High Mass YSO
}

\author{
L. J. Greenhill, M. J. Reid
}

Harvard-Smithsonian Center for Astrophysics, 60 Garden St, Cambridge, MA 02138, USA

C. J. Chandler

NRAO, P.O. Box O, Socorro, NM 87801, USA

\section{P. J. Diamond}

MERLIN/VLBI National Facility, Jodrell Bank Observatory, Macclesfield, SK11 9DL, UK

\author{
M. Elitzur \\ Department of Physics $\&$ Astronomy, University of Kentucky, \\ Lexington, $K Y$ 40506, USA
}

\begin{abstract}
High-mass star formation is not well understood chiefly because examples are deeply embedded, relatively distant, and crowded with sources of emission. Using VLA and VLBA observations of $\mathrm{H}_{2} \mathrm{O}$ and $\mathrm{SiO}$ maser emission, we have mapped in detail the structure and proper motion of material 20-500 AU from the closest high-mass YSO, radio source $I$ in the Orion KL region. We observe streams of material driven in a rotating, wide angle, bipolar wind from the the surface of an edge-on accretion disk. The example of source I provides strong evidence that high-mass star formation proceeds via accretion.
\end{abstract}

\section{Introduction}

High-mass star formation is poorly understood because theory has yet to resolve the balance of radiation pressure, gravity, magnetic energy, and thermal heating (e.g., McKee \& Tan 2002). Moreover, examples amenable to detailed study but young enough to have not yet formed HII regions are rare. First, massive YSOs are deeply embedded, form in clusters, and heat large amounts of surrounding dust and gas. As a result, the signatures of disks and outflows at small radii are easily lost in "background clutter." Second, regions of high-mass star formation are sufficiently distant $(z 500 \mathrm{pc})$ that infrared and radio observations of thermal gas and dust are often confusion-limited. Third, massive young stellar objects evolve rapidly, and by the time the surrounding medium is dispersed, accretion disk and outflow structures have been at least partly dispersed. In two of the best understood cases, G192.16-3.82 (Shepherd \& Kurtz 1999) and IRAS 20126+4104 (Zhang et al. 1998; Cesaroni et al. 1999), disk-like structures 
have been detected, but the rotation curves are barely resolved and the emission arises at large enough radii $(>1000 \mathrm{AU})$ that in depth study is difficult.

The infrared Kleinemann-Low (KL) nebula in Orion is the nearest $(\sim$ $500 \mathrm{pc}$ ), and most heavily studied region of high-mass star formation. The KL region is crowded, containing 16 identified mid-infrared peaks across $\sim 10^{4} \mathrm{AU}$ (Gezari, Backman, \& Werner 1998). Offset 0". 5 south of the prominent peak, IRc2, is radio source I, first detected by Churchwell et al. (1987). It has no infrared counterpart, but it does power compact distributions of $\mathrm{SiO}$ and $\mathrm{H}_{2} \mathrm{O}$ masers, and it has been identified as a probable deeply embedded, massive protostar or YSO (Gezari 1992; Menten \& Reid 1995).

The strongest $\mathrm{SiO}$ maser emission arises from the $\mathrm{v}=1$ vibrational state. Because of excitation requirements $\left(T>10^{3} \mathrm{~K}, n\left(\mathrm{H}_{2}\right) \sim 10^{10 \pm 1} \mathrm{~cm}^{-3}\right)$, it must originate close to the YSO. The first VLBI maps revealed an $\mathrm{X}$-shaped $\mathrm{SiO}$ emission locus extending outward 20-70 AU from source I. The $X$ also lay at the center of a $200 \times 600 \mathrm{AU}$ expanding patch of $\mathrm{H}_{2} \mathrm{O}$ masers distributed in two lobes each comprising red and blueshifted emission and bracketing source I (Greenhill et al. 1998; see also Gaume et al. 1998; Doeleman, Lonsdale, \& Pelkey 1999).

Taken alone, the distribution of $\mathrm{SiO}$ emission could have been interpreted equivalently in two ways. First, it could represent the turbulent limb of a highvelocity biconical outflow with a southeast-northwest axis. Second, it could represent hot material close to the top and bottom surfaces of an edge-on disk with a northeast-southwest rotation axis. Greenhill et al. and Doeleman et al. adopted the biconical outflow model, in part because the high-velocity outflow observed in the KL region on scales of $10^{4}$ AU (e.g., Allen \& Burton 1993; Schultz et al. 1999) subtended the opening angle of the putative $\mathrm{SiO}$ maser cone. In addition, Greenhill et al. (1998) noted that the distributions of proper motions and line-of-sight velocities among the surrounding $\mathrm{H}_{2} \mathrm{O}$ masers were not readily consistent with the edge-on disk model and proposed instead that the $\mathrm{H}_{2} \mathrm{O}$ masers lay on the surface of a nearly edge-on, inflating, equatorial doughnut-like shell. This shell could arise from a slow stellar equatorial wind advancing into the surrounding medium or from photo-evaporation of an accretion disk. Because the major axis of this shell was aligned with the the so-called " $18 \mathrm{~km} \mathrm{~s}^{-1}$ " outflow in the KL region (e.g., Genzel \& Stutzki 1989), it appeared that source I might drive the two most prominent outflows in the region.

Despite circumstantial evidence tying source I to large scale outflows in the KL region, ambiguity has remained. On the one hand, Menten \& Reid (1995) noted that another infrared source, $n$, lies closer to the previously estimated center of the flows (Genzel et al. 1981). Moreover, high-velocity gas far to the northwest is blueshifted, while the corresponding outflow cone near source $I$ is redshifted. On the other hand, the compact bipolar radio lobes of source $\mathrm{n}$ are oriented north-south, $40-50^{\circ}$ from the principal axes of the low and high-velocity large scale flows in the $\mathrm{KL}$ region.

To better understand the KL region and to test the biconical outflow model proposed for source I, we re-mapped the $\mathrm{SiO}$ and $\mathrm{H}_{2} \mathrm{O}$ maser emission, covering three $J=1 \rightarrow 0$ transitions of $\mathrm{SiO}(\mathrm{v}=0,1, \& 2)$ and the full velocity range of $\mathrm{H}_{2} \mathrm{O}$ emission. (The original maps presented by Greenhill et al. were significantly limited in dynamic range, especially at velocities close to the systemic velocity.) We measured the proper motion of the $\mathrm{SiO} v=1 \& 2$ emission, observ- 
ing monthly over $\sim 3.5$ years, which is $\sim 30 \%$ of the dynamical crossing time of the outflow. Here we discuss proper motions over a four month interval.

\section{Observations and Data Reduction}

We observed the $\mathrm{v}=1 \& 2 J=1 \rightarrow 0$ transition of $\mathrm{SiO}$ with the VLBA, and the $\mathrm{v}=0 \mathrm{~J}=1 \rightarrow 0$ transition of $\mathrm{SiO}$ and $6_{16} \rightarrow 5_{23}$ transition of $\mathrm{H}_{2} \mathrm{O}$ with the VLA in its largest configuration and including the nearby Pie Town VLBA antenna. We obtained angular resolutions of $\sim 0.2$ milliarcseconds (mas) with the VLBA and $\sim 50$ mas with the VLA. The spectral channel spacings in the VLBA and VLA imaging were $\sim 0.4 \mathrm{~km} \mathrm{~s}^{-1}$ and $\sim 2.6 \mathrm{~km} \mathrm{~s}^{-1}$, respectively.

Using the VLBA, we observed the $\mathrm{SiO} \mathrm{v}=1 \& 2$ emission (from $V_{\mathrm{LSR}} \sim-15$ to $+25 \mathrm{~km} \mathrm{~s}^{-1}$ ) simultaneously to enable registration of both lines to $\sim 0.1$ mas. Because emission from source I itself is thermal, it could not be detected with the VLBA. To locate the position of the YSO on the VLBA maps, we convolved the maps with a circular beam comparable in size to the beam of the VLA at $\lambda 7 \mathrm{~mm}$ and overlayed the degraded VLBA map on the VLA map of Menten \& Reid (1995), who detected and registered the $\mathrm{SiO} v=1$ and thermal continuum emission of the YSO. The uncertainty in this registration is $\sim 10$ mas.

Using our VLA observations, we measured the positions of the $\mathrm{SiO} v=0$ masers $\left(\sim-10\right.$ to $\left.+22 \mathrm{~km} \mathrm{~s}^{-1}\right)$ with respect to the $\mathrm{v}=1$ emission, which was observed simultaneously so as to provide an astrometric reference. The uncertainty in position relative to source $\mathrm{I}$ is $\sim 10$ mas. The positions of the $\mathrm{H}_{2} \mathrm{O}$ masers $\left(\sim-10\right.$ to $\left.+16 \mathrm{~km} \mathrm{~s}^{-1}\right)$ were measured with respect to a nearby quasar and compared to the absolute position of source I (Menten \& Reid 1995). The uncertainty in this comparison is 30 mas.

\section{Results and Discussion}

Our more extensive mapping of the $\mathrm{SiO}$ and $\mathrm{H}_{2} \mathrm{O}$ maser emission now supports the edge-on disk model previously discarded. We propose that the $\mathrm{v}=1 \& 2 \mathrm{SiO}$ maser emission traces material streaming in a rotating funnel-like flow from the upper and lower surfaces of an accretion disk of a massive YSO. The $\mathrm{v}=0 \mathrm{SiO}$ and $\mathrm{H}_{2} \mathrm{O}$ masers lie in a bipolar outflow along the disk rotation axis.

\section{1. $R<70 \mathrm{AU}$}

In the most recent observations, the $\mathrm{v}=1 \& 2 \mathrm{SiO}$ emission traces an $\mathrm{X}$ as before (Figure 1), but we note two important new details. First, there is a "bridge" of maser emission extending from the base of the south arm to the base of the west arm, and there is a gradient in line-of-sight velocity along the bridge. Second, the arms are not radial. These new findings may be consequences of our having now mapped the $\mathrm{v}=2$ as well as $\mathrm{v}=1$ emission. (For instance, the bridge is outlined principally by $\mathrm{v}=2$ emission.) Both findings are difficult to explain in the context of the biconical flow model. However, they are readily explained in the context of the edge-on disk model. The bridge and associated velocity gradient are natural signatures of emission from the front side of a rotating disk that is tipped down slightly to the southwest. The canting of the arms, so that 
they are not radial, is also suggestive of reflection symmetry about a plane (i.e., disk).
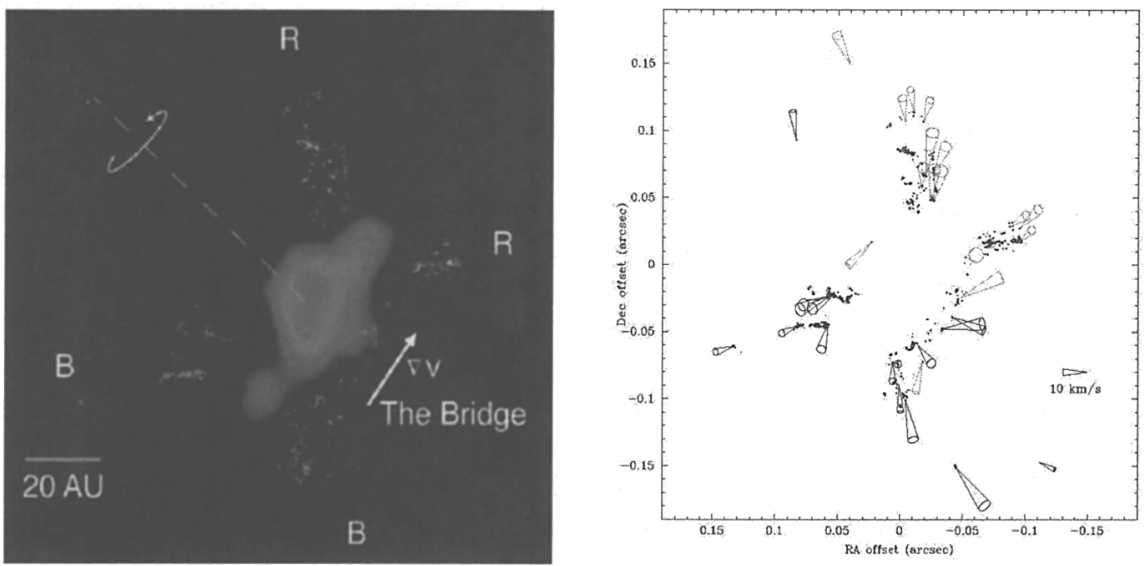

Figure 1. (Left:) Vibrationally excited SiO masers forming an $\mathrm{X}$ and $\lambda 7 \mathrm{~mm}$ continuum emission (grayscale) observed by Menten \& Reid (in prep). Emission redshifted (R) with respect to the systemic velocity $\left(V_{\mathrm{LSR}}=5 \mathrm{~km} \mathrm{~s}^{-1}\right)$ lies to the north and west. Blueshifted emission (B) is opposite. The line emission probably originates in rotating high density material driven from the surface of an edge-on disk. The continuum probably arises at least in part from $\mathrm{H}^{-}$free-free disk emission. (Right:) Proper motions of $\mathrm{v}=1 \& 2 \mathrm{SiO}$ maser clumps (Chandler et al., in prep). The lengths of the cones indicate $3-\mathrm{D}$ velocities. The aspects of the cones indicate inclinations with respect to the line of sight. Blueshifted motions are black, redshifted are gray.

In order to estimate the proper motions of maser clumps, we identified one in each quadrant of the $X$ that persisted in each epoch and maintained the same line-of-sight velocity. We overlayed images for the four epochs and registered them to achieve zero mean proper motion for the source as a whole. Most proper motions are 10 to $15 \mathrm{~km} \mathrm{~s}^{-1}$, though some clumps are nearly stationary on the sky. (One $\mathrm{km} \mathrm{s}^{-1}$ over four months corresponds to $\sim 1$ VLBA beamwidth.) The maximum observed 3-D velocity is $\sim 23 \mathrm{~km} \mathrm{~s}^{-1}$. The proper motions of maser clumps are chiefly along the four arms of the $X$ (Figure 1). In the bridge, the motions are both outward and tangential, indicative of rotation and strongly in support of the edge-on disk model (Chandler et al., in prep).

We suggest that the arms of $\mathrm{SiO}$ emission represent the limbs of a bipolar funnel-like outflow (Figure 2), probably the turbulent shocked interface between outflowing and accreting material. In a rotating system, strong maser emission is expected along the limbs because that is where the longest maser gain paths lie owing to projection effects. Additional emission from the bridge may mark the nearside of the outflow wall, where at the base, higher densities or temperatures could compensate for otherwise shorter gain paths. The dynamical mass of the YSO is difficult to estimate because the maser material is not in simple Keplerian rotation. If the observed 3-D velocity is greater than the escape velocity, then 
the enclosed mass is $\gtrsim 6 \mathrm{M}_{\odot}$, for a radius of $25 \mathrm{AU}$ and velocity of $25 \mathrm{~km} \mathrm{~s}^{-1}$. We note that the 3-D motion of the maser material is helical. This may indicate the presence of a strong magnetic field that is probably anchored to the accretion disk, since massive stars are radiative and may not generate their own field. If the magnetic and kinetic energy densities are of the same order, then the field is on the order of $1 \mathrm{G}$, for a gas density of $10^{10} \mathrm{~cm}^{-3}$.

\section{2. $\quad R>70 \mathrm{AU}$}

The $\mathrm{v}=0 \mathrm{SiO}$ and $\mathrm{H}_{2} \mathrm{O}$ maser emission arises chiefly from two "polar caps" that subtend the $\sim 90^{\circ}$ opening angle of the outflow along the disk rotation axis (Figure 2). The velocity structure of the emission is somewhat disorganized and difficult to model in detail. However, the two lobes (northeast and southwest) display the same ranges of velocity, indicative of outflow in the plane of the sky. Within each lobe, the bulk of the emission displays a red-blue asymmetry across the rotation axis in the same sense as the disk rotation close to source I, although further study is required. Otherwise, it may signify coupling between the velocity field downstream in the outflow and the underlying accretion disk, possibly via magnetic processes.

Substantial overlap of $\mathrm{H}_{2} \mathrm{O}$ maser and $\mathrm{SiO} v=0$ emission along the line of sight is surprising because maser action in each species requires quite different densities, $10^{8-10} \mathrm{~cm}^{-3}$ vs $10^{5-6} \mathrm{~cm}^{-3}$, respectively. Moreover, because molecule reformation on dust grains is essential for $\mathrm{H}_{2} \mathrm{O}$ maser action behind shocks, gas phase $\mathrm{SiO}$ would be depleted. In principle, the $\mathrm{H}_{2} \mathrm{O}$ and $\mathrm{v}=0 \mathrm{SiO}$ maser volumes could be intermingled if the flow were inhomogeneous and if shock induced grain sputtering enhanced gas phase concentrations of SiO. However, the requisite $>10^{2}$ density contrast between emitting regions would be difficult to explain in the apparent absence of high-velocity $\left(\gg 50 \mathrm{~km} \mathrm{~s}^{-1}\right)$ gas motions.

We suggest that the wall of the outflow traced by $\mathrm{v}=1 \& 2 \mathrm{SiO}$ masers at radii $<70 \mathrm{AU}$ extends to larger radii where it supports $\mathrm{H}_{2} \mathrm{O}$ maser emission. The outflow itself has low enough density to support the $\mathrm{v}=0 \mathrm{SiO}$ maser emission, which lies along the same line of sight as the $\mathrm{H}_{2} \mathrm{O}$ emission in projection. The ordered velocity structure of the $\mathrm{v}=1 \& 2 \mathrm{SiO}$ maser emission and the relatively complicated velocity structure of the $\mathrm{H}_{2} \mathrm{O}$ and $\mathrm{v}=0 \mathrm{SiO}$ maser emission at larger radii may be a consequence of the increased effects turbulence or collisions with inflowing or ambient material downstream in the outflow.

\section{Summary}

We have fully resolved the structure and dynamics of material at radii of 20$500 \mathrm{AU}$ from radio source I in the Orion KL region. Our maps provide the most detailed picture yet of molecular material so close to a massive YSO. We propose a new model for source I in which the accretion disk is edge-on and a wide-angle wind feeds a funnel-like rotating bipolar outflow. Consequently, the case of source I provides strong evidence that accretion in high-mass star formation proceeds via orderly disk-mediated accretion as opposed to coalescence of low mass stars (Bonnell et al. 1998). 

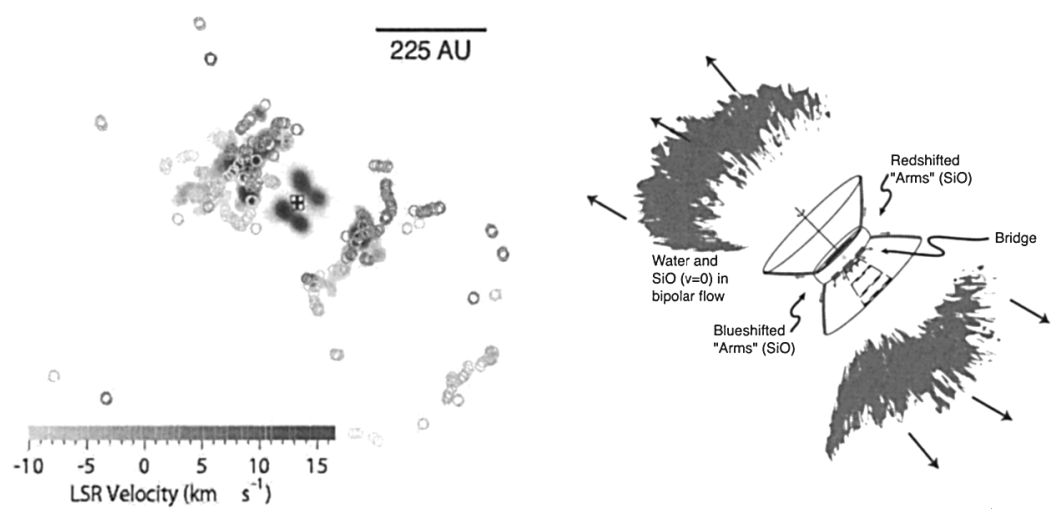

Figure 2. (Left:) Superposition of $\mathrm{SiO}$ maser emission integrated in velocity (grayscale) and $\mathrm{H}_{2} \mathrm{O}$ maser emission (circles). The "cloverleaf" near source $\mathrm{I}$ (the box and cross) is $\mathrm{v}=1 \& 2 \mathrm{SiO}$ emission. The arcs to the northeast and southwest are $\mathrm{v}=0$ emission. The $\mathrm{H}_{2} \mathrm{O}$ spots mark the emission centroids in the spectral channels. Redshifted emission is gray, blueshifted is black. (Right:) Sketch of the proposed edge-on disk model, indicating the loci of $\mathrm{SiO}$ and $\mathrm{H}_{2} \mathrm{O}$ maser emission.

\section{References}

Allen, D. A., \& Burton, M. G. 1993, Nature, 363, 54

Bonnell, I. A., Bate, M. R., \& Zinnecker, H. 1998, MNRAS, 298, 93

Cesaroni, R., et al. 1999, A\&A, 345, 949

Churchwell, E., Wood, D. O. S., Felli, M., \& Massi, M. 1987, ApJ, 321, 516

Doeleman, S. S., Lonsdale, C. J., \& Pelkey, S. 1999, ApJ, 510, L55

Gaume, R. A., Wilson, T. L., Vrba, F. J., Johnston, K. J., \& Schmid-Burgk, J. 1998, ApJ, 493, 940

Genzel, R., \& Stutzki, J. 1989, ARAA, 27, 41

Gezari, D. Y. 1992, ApJ, 396, L43

Gezari, D. Y, Backman, D. E., \& Werner, M. W. 1998, ApJ, 509, 283

Greenhill, L. J., Gwinn, C. R., Schwartz, C., Moran, J. M., \& Diamond, P. J. 1998, Nature, 396, 650

McKee, C. F., Tan, J. C. 2002, Nature, 416, 59

Menten, K. M., \& Reid, M. J. 1995, ApJ, 445, L157

Plambeck, R. L., Wright, M. C. H., Mundy, L. G., Looney, L. W. 1995, ApJ, 455, L189

Schultz, A. S. B, et al. 1999, ApJ, 511, 282

Shepherd, D. S., \& Kurtz, S. E. 1999, 523, 690

Zhang, Q., Hunter, T. R., \& Sridharan, T. K. 1998, ApJ, 505, L151 